\title{
BMJ Open Is microdiffusion imaging able to improve the detection of cervical myelopathy? Study protocol of a prospective observational trial (MIDICAM-Trial)
}

\author{
Marc Hohenhaus, ${ }^{1}$ Karl Egger, ${ }^{2}$ Jan-Helge Klingler, ${ }^{1}$ Ulrich Hubbe, \\ Marco Reisert, ${ }^{3}$ Katharina Wolf ${ }^{4}$
}

To cite: Hohenhaus $\mathrm{M}$,

Egger K, Klingler J-H, et al. Is microdiffusion imaging able to improve the detection of cervical myelopathy? Study protocol of a prospective observational trial (MIDICAM-Trial). BMJ Open 2019;9:e029153. doi:10.1136/ bmjopen-2019-029153

- Prepublication history for this paper are available online. To view these files, please visit the journal online (http://dx.doi. org/10.1136/bmjopen-2019029153).

Received 14 January 2019 Revised 11 July 2019 Accepted 01 August 2019
D) Check for updates

(c) Author(s) (or their employer(s)) 2019. Re-use permitted under CC BY-NC. No commercial re-use. See rights and permissions. Published by BMJ.

For numbered affiliations see end of article.

\section{Correspondence to}

Dr Marc Hohenhaus; marc.hohenhaus@uniklinikfreiburg.de

\section{ABSTRACT}

Introduction The diagnosis of degenerative cervical myelopathy (DCM) is difficult in numerous patients due to the limited correlation of clinical symptoms, electrophysiology and MRI. This applies especially for early disease stages with mild symptoms or in uncertainty due to comorbidities. Conventional MRI myelopathy signs show a restricted sensitivity to clinical symptoms of at most $60 \%$. It is desirable to select patients for surgical treatment as early as possible before irreversible neurological damage occurs. To improve treatment, a more reliable imaging is necessary. Microdiffusion imaging (MIDI) is an innovative MRI modality to depict tissue alterations within one voxel based on diffusionweighted imaging (DWI) postprocessing. By separating the affected area into several mesoscopic compartments, pathological changes might be detected more sensitive through this subtle tissue resolution. We hypothesise, that MIDI shows myelopathic alterations more sensitive than conventional MRI and improves the correlation to functional impairment.

Methods and analysis In this prospective, observational trial, 130 patients with a relevant degenerative cervical spinal stenosis receive MRI including MIDI and a standard clinical and electrophysiological assessment. Special subvoxel diffusion parameters are calculated. Clinical follow-ups are conducted after 3, 6 and with additional MRI and electrophysiology after 12 months. The primary endpoint is the sensitivity of MIDI to detect functional myelopathy defined by clinical and electrophysiological features correlated to conventional MRI myelopathy signs. Twenty healthy subjects will be included as negative control. The results will provide new insights into the development of mesoscopic spinal cord alterations in DCM associated to the clinical course. Aim is to improve the diagnostics of incipient myelopathy through this new modality.

Ethics and dissemination The study protocol is approved by the Ethics Committee of the University of Freiburg (reference 261/17). The results will be published in a peerreviewed journal.

Trial registration number DRKS00012962.
Strengths and limitations of this study

- This will be the first prospective trial to clarify the diagnostic uncertainty in patients with suspected cervical myelopathy by using a new MRI diffusion modality with a special subvoxel analysis ('microdiffusion imaging', MIDI).

- The evaluation of all severity levels of myelopathy and 1-year clinical, electrophysiological and imaging follow-up will provide new insights into the natural and postoperative course of affected patients and thus increase diagnostic predictability.

- MIDI is expected to be the current state-of-the-art imaging modality for cervical myelopathy with a higher sensitivity compared with conventional $\mathrm{T} 1$ and T2 MRI with only approximately $60 \%$.

- A limitation is the still existing lack of 'gold standard' for the diagnosis of cervical myelopathy leaving some uncertainty for an evaluation of a new diagnostic method which is tried to fix through a combined clinical and electrophysiological definition.

- The expected MIDI-based improvements in diagnosis and therapeutic decision making, especially in early stages of cervical myelopathy, will lead to further investigations with longer follow-up and randomised treatment protocols.

\section{INTRODUCTION}

The incidence of degenerative cervical myelopathy (DCM) is high with 4-8 per 100 000 inpatient treatments per year. ${ }^{1}$ Due to this frequency and high long-term morbidity, DCM is of great social and health economic relevance. ${ }^{2}$ In older patients, it is the most frequent cause of spinal cord injury. ${ }^{2}$ Surgical treatment is recommended in patients with definite clinical cervical myelopathy due to degenerative spinal cord compression and an early decompression saves neurological function. ${ }^{1}$ So the early detection of patients likely 
to develop myelopathy is necessary to avoid persistent deficits.

The indication for surgical treatment in suspected DCM is supported by clinical, electrophysiological and imaging aspects. The clinical detection of only mild myelopathic symptoms, especially in early stages of the disease, can be difficult, but poses the challenge, since the development of further neurological alterations can be prevented with a significant better prognosis. The duration of symptoms is a strong predictor for a worse outcome. ${ }^{3}$ Furthermore, the typical symptoms as ataxic gait disorders, fine motor dysfunction or perceptive abnormalities can also be generated by different co-morbidities, especially in older patients who are primarily affected.

The usual electrophysiological diagnostics can also not provide sufficient safety in mild affected patients. ${ }^{4-6}$ The evidence is heterogeneous without clear examination guidelines for a reliable detection of affected patients. Holly et al reported a prognostic power of reduced somatosensory-evoked potentials (SSEP), especially of the median nerve. ${ }^{6}$ Motor-evoked potentials (MEP) and transcranial magnetic stimulation should still show the best correlation with primary clinical findings, even though the exact diagnostic and prognostic value has not yet been investigated. ${ }^{4}$ Electromyography (EMG) alterations in patients with an asymptomatic cervical stenosis seem to be associated with a subsequent development of a manifest myelopathy. ${ }^{7}$ Contact heat-evoked potentials which might currently show the best sensitivity for a cervical myelopathy, are not yet a clinical standard procedure. ${ }^{8}$

MRI is the gold standard for the depiction of cervical degenerative spinal disorders due to its high lesion perceptibility and local resolution. ${ }^{9} 10$ Signal alterations like T1-weighted hypointense areas and T2-weighted (T2w) hyperintensities are widely accepted as 'myelopathy signs' and associated with a poor neurological function before and after treatment. ${ }^{11-13}$ But in primary diagnosis these signs have a limited value too. The correlation with clinical symptoms is described with a sensitivity of at most $60 \%{ }^{1314}$ At present, the indication for surgical decompression based on a cervical spinal canal stenosis with intramedullary T2w hyperintensity irrespective of clinical deficits, is controversially discussed. Even more aggressive surgical strategies (eg, based on isolated radiographic spinal canal stenosis without functional or radiographic myelopathy signs) are not recommended because of a low risk to develop clinical alterations and therefore a worse benefit-risk ratio receiving surgery. ${ }^{15}$ Surgical complications are reported in $11 \%-38 \% .^{16}$

Also, the prediction of the individual prognosis is not yet reliable based on MRI or electrophysiological findings due to the heterogeneous results of the available studies. ${ }^{11-13} 17-20$

In conclusion, the sensitivity of all three diagnostic tools increases with the severity of the disease and is less reliable in early stages. A definite clinical syndrome with certain electrophysiological results of cervical myelopathy and MRI myelopathy signals usually leads to an uncontroversial indication for surgical decompression. However, in our experience, many patients do not show such a conclusive configuration.

As a consequence, a more reliable diagnostic method for detection of cervical myelopathy is necessary. For this aim, we developed the microdiffusion imaging (MIDI), based on a post-processing method of DWI. The modality enables to detect mesoscopic tissue alterations in a subvoxel volume. ${ }^{21}$ With help of this complex post-processing through a supervised machine learning algorithm, it is possible to divide the examined spinal cord area into several subcompartments within one pixel. ${ }^{21}$ The interesting advancement is the creation of intra-axonal and extra-axonal spaces with its own water distributions and diffusion parameters. This microstructural resolution can reflect more pathophysiological aspects of incipient spinal cord injuries. ${ }^{22}$ The previous application of this new MRI modality was in ischaemic stroke patients, where a higher sensitivity of early ischaemic altered cerebral areas could be demonstrated in comparison with conventional DWI. ${ }^{21}$

Our goal is to establish a more sensitive imaging for the detection of myelopathy in the cervical spinal cord. Furthermore, we want to correlate our MRI findings with the clinical course and develop new prognostic parameters for patients with DCM. With the help of this new, routinely applicable MRI modality, a better separation of patients with and without myelopathy with regard to surgical treatment is to be achieved.

\section{METHODS AND ANALYSIS}

\section{Study design}

This study is a controlled, prospective, longitudinal, monocentric, observational trial. A signed informed written consent will be obtained from each participant.

\section{Patient and public involvement}

This is an investigator-initiated trial out of the clinical experience to address the frequent problem of an unclear myelopathy in patients with degenerative cervical spine disease. Patients or public were not involved in the preparation of the study design. The results will be disseminated to the participants in periodical local patient information events and if requested in a written form including the individual parameters and whole study results after finishing the trial.

\section{Study population}

On target are patients ( $\mathrm{n}=130)$ with cervical disc herniation and contact to the spinal cord or relevant degenerative spinal canal stenosis. A second population of healthy volunteers $(n=20)$ will be included as age-matched and gender-matched control group for the new imaging modality.

\section{Inclusion and exclusion criteria}

Patients over 18 years with symptoms of a degenerative cervical spine disease and radiographic cervical disc 


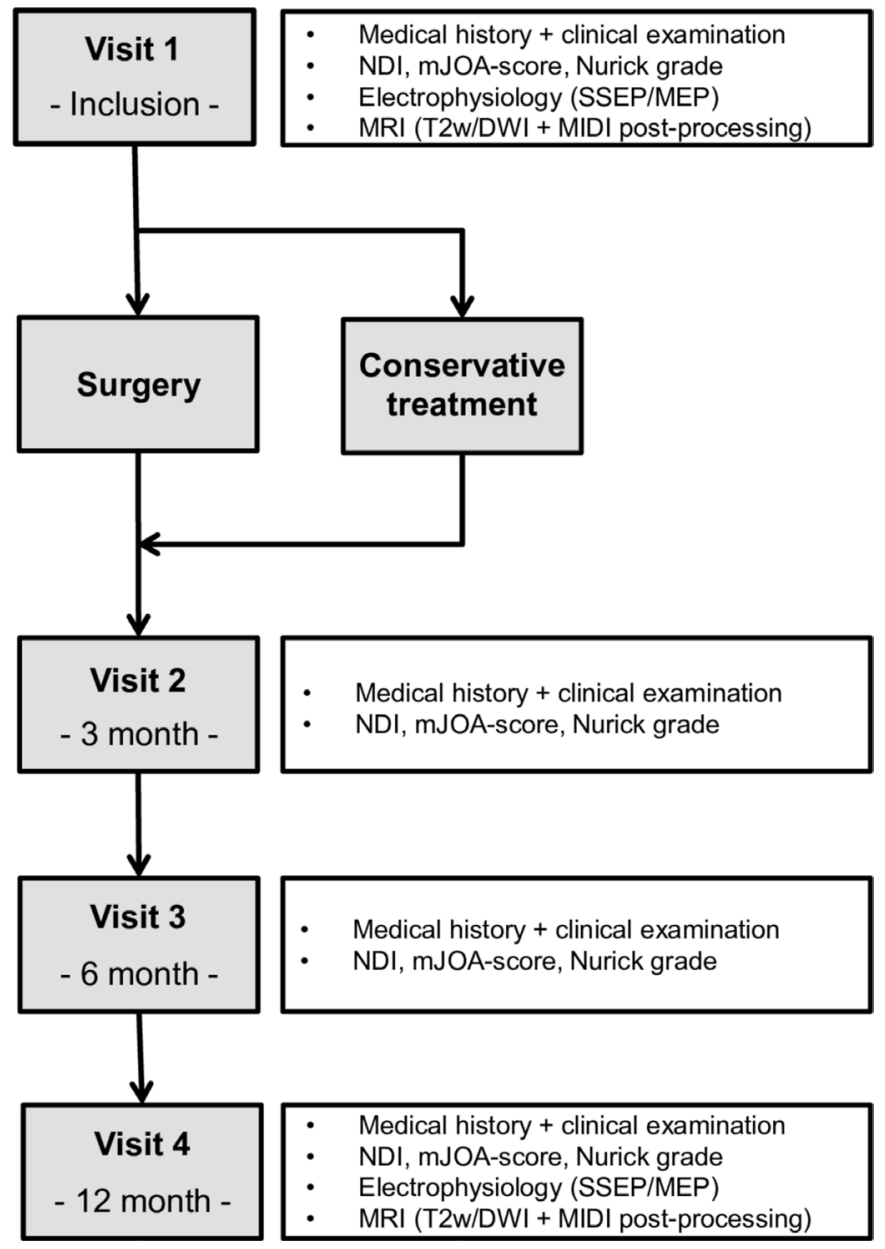

Figure 1 Flow chart of the trial timeline after patient inclusion. DWI, diffusion-weighted imaging; MEP, motorevoked potentials; MIDI, microdiffusion imaging; mJOA, modified Japanese-Orthopaedic-Association-score; NDI, Neck Disability Index; SSEP, somatosensory-evoked potentials.

damage with contact to the spinal cord or spinal canal stenosis with spinal cord compression will be included. The compression should be primarily at a singular level.

Exclusion criteria are any contraindication for MRI, previous major operations of the cervical spine as well as non-degenerative causes of the associated alterations (eg, tumours, inflammation/infection and trauma). Multisegmental relevant spinal cord compression will be excluded. Additionally all patients with severe comorbidity are excluded from the study. Controls are required to have no neurological disease and no severe comorbidity.

\section{Recruitment procedure}

Patients presenting with symptoms of a degenerative cervical spine disease at the outpatient clinics of the Departments of Neurosurgery and Neurology at the University Medical Center Freiburg are screened. If eligible without meeting any exclusion parameters and once written consent is obtained, the patients receive their first appointments at our Departments in context of the study. Controls are recruited by advertisements on the clinic's black board and within the weekly online-newsletter.

\section{Trial timeline}

All included subjects receive a full clinical, electrophysiological and MRI work-up within twoweeks after enrolment into the study. The conservative or surgical therapy strategy is determined with the patient independently to the study corresponding to the current German guidelines. ${ }^{23}$ All patients, conservative and surgical branch, receive clinical follow-ups after three, six and twelve months. At twelve month, MRI with MIDI and electrophysiological assessments are repeated. An overview of the trial timeline is shown in figure 1.

\section{Clinical parameters}

The epidemiological and symptom characteristics as well as the neurological examination results are documented on a specified protocol. The pain localisation and intensity in combination with the associated limitations of the daily life are recorded by the Numeric Rating Scale, Neck Disability Index and Karnofsky performance status scale. The state of painkiller intake is documented regarding to the WHO classification. The neurological examination is done according to the International Standards for Neurological Classification of Spinal Cord Injury with evaluation of motoric deficits due to dermatomal characteristic muscles graded in strength from zero to five according to the British Medical Research Council and reflex status in three grades: attenuated, regular and enhanced. The sensory alterations are documented as radicular or unspecific for each limb. According to the work of Zhong et al the foot tapping test is performed for each leg. ${ }^{24}$ The severity of clinical myelopathy is rated by the modified Japanese-Orthopaedic-Association-score (mJOA) and Nurick grade. ${ }^{25} 26$

\section{Electrophysiological parameters}

Only patients receive electrophysiological work-up. Standard normal values are applied as control parameter. For the evaluation of cervical myelopathy, the measurement standards with SSEP of the tibial and median nerve (including lumbal, erb and C7 interpeak latencies) and MEP to the musculus tibialis anterior and the musculus abductor digiti minimi are performed. ${ }^{27}$ The approximated central motoric latency is calculated by subtraction of additionally single-pulse nuchal and lumbar MEP of the cortical MEP to the arm and leg, respectively. ${ }^{28}$ If individually indicated by an independent neurologist, further assessments (eg, EMG or electroneurography) are performed for other peripheral or central neurological co-morbidities or radicular involvement. $^{27}$

The results of the evoked potentials are divided into four grades: normal (3), mildly deteriorated (2), severely deteriorated (1) and abolished (0) adapted to Kuhn et al and Petersen et $a l^{2930}$ 


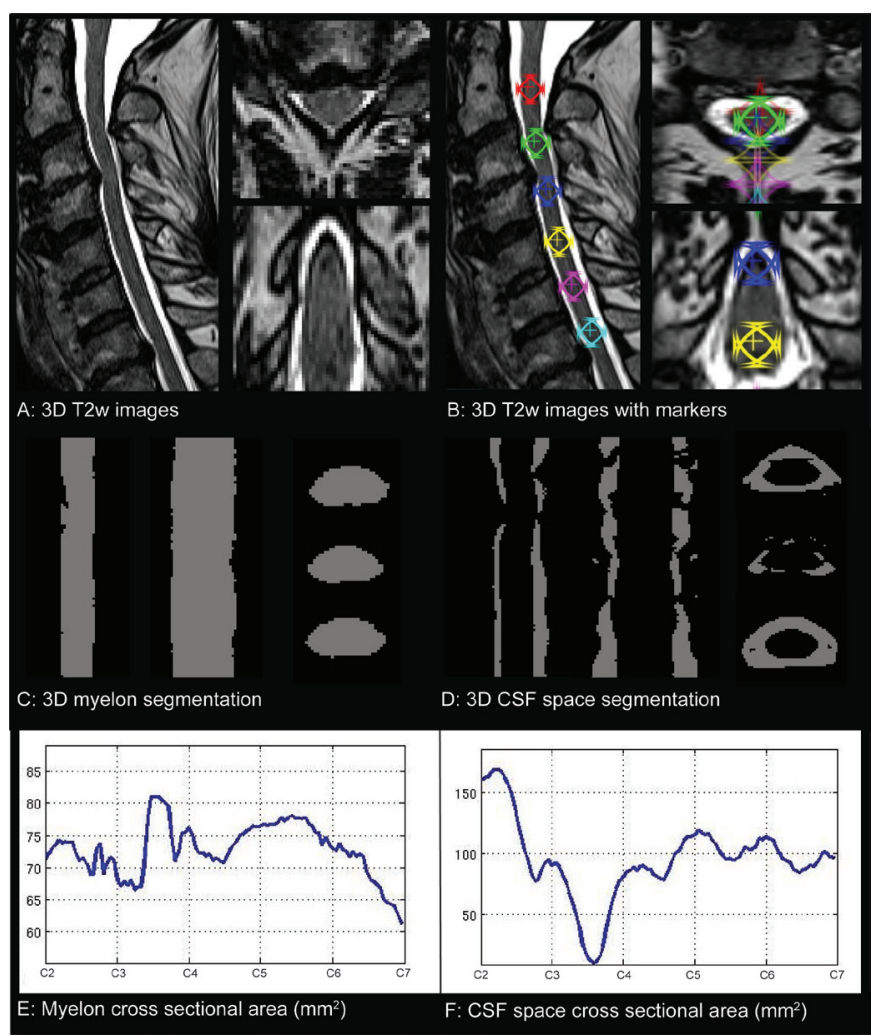

Figure 2 Overview of the automated segmentation process for myelon and spinal canal. The MRIs of a patient with cervical stenosis in C3/4 are shown in all three dimensions. (A) Standard high-resolution 3D T2w images. (B) T2w images with manual placed markers on the myelon centred behind vertebral body 2-7 as definition of the anatomic cervical level. This is the only manual application within the process. (C and D) Automated 3D myelon and CSF space segmentation from marker $\mathrm{C} 2-\mathrm{C} 7$. The total of both defines the whole spinal canal area. (E and $F$ ) Absolute value curves for myelon and CSF space cross-sectional area in $\mathrm{mm}^{2}$ from C2-C7. $3 \mathrm{D}$, three dimensional; CSF, cerebrospinal fluid; T2w, T2weighted.

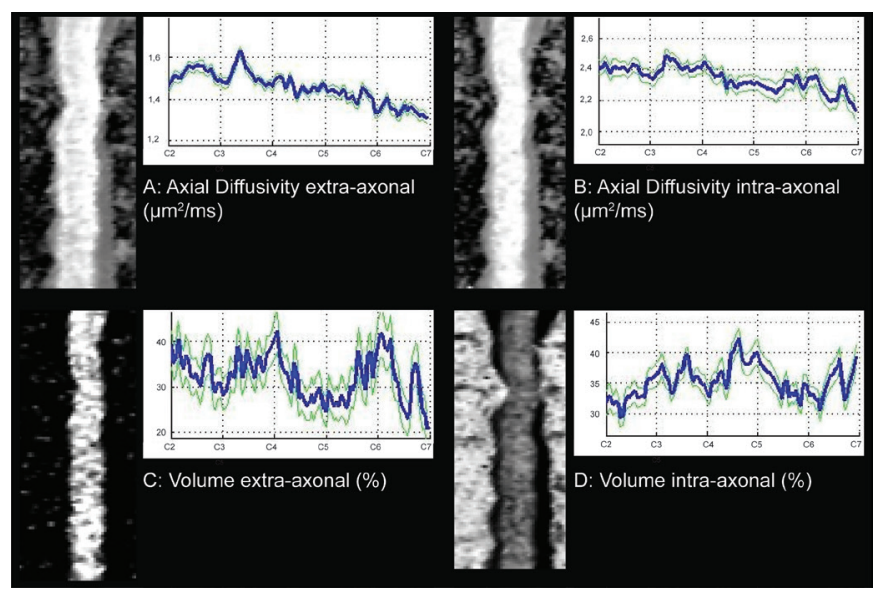

Figure 3 The related sagittal planes as well as value curves for extra-axonal (A) and intra-axonal (B) diffusivity in $\mu \mathrm{m}^{2} / \mathrm{ms}$ and extra-axonal $(C)$ and intra-axonal (D) volume as fraction in percent.
Table 1 Recorded MRI parameters for evaluation of the spinal canal diameters and associated medullary myelopathic signal changes

\begin{tabular}{|c|c|}
\hline T2w & MIDI \\
\hline $\begin{array}{l}\text { Anteroposterior diameter* } \\
\text { Transverse diameter } \\
\text { Spinal cord circumference* } \\
\text { Cross-sectional area* } \\
\text { Compression ratio† } \\
\text { Medullary T2 } \\
\text { hyperintensity } ¥ \\
\text { Medullary signal intensity } \\
\text { ratio† }\end{array}$ & $\begin{array}{l}\text { Volume intra-axonal/extra- } \\
\text { axonal }^{*} \\
\text { Axial diffusivity intra-axonal/ } \\
\text { extra-axonal } \\
\text { Radial diffusivity extra-axonal* } \\
\text { Volume cerebrospinal fluid* }\end{array}$ \\
\hline
\end{tabular}

*Measurement of the spinal cord and bony spinal canal for each level of the cervical spine from C2 to C7 including the area of maximal compression.

†Ratios of maximum stenotic areas to adjacent levels of the spinal canal and spinal cord above and below.

$\ddagger$ Appearance of the signal evaluated by two independent raters with calculation of the interrater reliability.

MIDI, microdiffusion imaging; T2w, T2-weighted.

\section{MRI parameters}

All images are generated with a clinical 3 Tesla MRI scanner (SIEMENS Magnetom Prisma). Primarily a standardised $\mathrm{T} 2 \mathrm{w}$ three-dimensional sequence in sagittal plane is made with the following parameters: voxel size 0.6x 0.6x $1.0 \mathrm{~mm}$, TR $1500 \mathrm{~ms}$, TE $134 \mathrm{~ms}$, Flip angle $105^{\circ}$, GRAPPA PAT: 3, acquisition time 3:53 min.

The whole cervical spinal cord is segmented by an automatic algorithm on the T2w images from $\mathrm{C} 2$ to $\mathrm{C} 7$ (figure 2). The spinal canal diameter in the sagittal and reconstructed transverse $\mathrm{T} 2 \mathrm{w}$ images at the maximal compressed segment and at the surrounding unaffected areas cranial and caudal of the stenosis is measured. ${ }^{31}$ The isolated area of the spinal cord is separated similarly (figure 2). The compression ratios for spinal cord and canal are calculated according to Kadanka et al. ${ }^{31} \mathrm{We}$ generate absolute value curves for the whole depicted cervical spine for every acquired parameter like shown in figure $2(\mathrm{E}+\mathrm{F})$ and figure 3 . The presence of a T2w hyperintense myelopathy sign is subjectively determined by two independent raters and additionally measured as signal intensity ratio between the affected and normal spinal cord. All collected parameters are summarised in table 1.

The second imaging step is the acquisition of the DWI as basis for the calculation of the MIDI values with the following sequence parameters: SIEMENS RESOLVE, voxel size 1.0x 1.0x 2.5 mm, TR $2600 \mathrm{~ms}$, TE1 $66 \mathrm{~ms}$, TE 2 $100 \mathrm{~ms}$, flip angle $180^{\circ}$, GRAPPA PAT: 2, acquisition time 15:04 min, 40 diffusion directions distributed hexagonally within a ball in q-space with following b-values: 400, 1200, $1600,2000 \mathrm{~s} / \mathrm{mm}^{2}$.

The DWIs are analysed with the Bayesian approach described in Reisert et al to obtain the innovative microdiffusion parameters shown in table $1 .^{21}$ This is based on a three compartment model: intra-axonal tissue, extra-axonal tissue and free water. Besides the tissue fractions, 
also the diffusion parameters of each compartment are estimated (see table 1). Before analysis, the DWIs are corrected for motion, Gibbs-ringing artefact and denoised. $^{32}{ }^{33}$ All computation is conducted within the NORA framework (www.nora-imaging.org) through a specialised in-house software pipeline.

Concerning the evaluation of myelopathy, the different parameters are compared between spinal cord areas in the field of the stenosis with the cranial and caudal located healthy regions (figure 3). The spinal cord is therefore separated automatically in the same algorithm like mentioned above.

\section{Pathophysiological background}

The pathophysiological equivalent of the different MIDI parameters are derived from the evolution of a degenerative myelopathy with hypoxia due to chronic compression, interruption of the blood-spinal cord barrier, following cellular dysfunction with cytotoxic oedema, up to tissue necrosis and spinal cord atrophy. ${ }^{2234}$ Basis for those calculations is a biophysical model creating three compartments: intra-axonal and extra-axonal space and the free water compartment. ${ }^{21}$ Measured are local diffusivities and volume fractions in the several compartments. An isolated demyelination might cause a relatively increased extra-axonal volume. The development of a cytotoxic oedema with intracellular directed diffusion processes, changes the intra-axonal and extra-axonal water distribution. Associated local alterations of the axial and radial diffusivity are already known from cerebral ischaemia. The intra-axonal axial diffusivity might get more undirected. As effect of the local microperfusion deficit, the axonal beading might induce similar changings. With this high-resolution diffusion images we want to detect the early pathophysiological alterations before occurrence of focal necrosis and spinal cord atrophy, depicted on $\mathrm{T} 2 \mathrm{w}$ images as mostly irreversible injury. ${ }^{34} 35$

\section{Endpoint measurement}

The primary endpoint of our study examines the accuracy of conventional T2w imaging compared with the novel MIDI parameters based on the sensitivity of both methods to detect a functional myelopathy. Secondary endpoints will investigate the specificity as well as the positive and negative predictive value.

Due to the fact that there is no generally accepted definition of myelopathy in current literature and guidelines, we define 'functional myelopathy' as mJOA-score $\leq 15$ in combination with an at least mildly deteriorated ('grade 2') SSEP of the median nerve or prolonged cervical spinal interpeak-latency. According to our definition, we group our patients into functional present or absent myelopathy, in order to validate our imaging modalities.

Further we evaluate the course of the disease for 1 year with an additional follow-up MRI and electrophysiology. The MIDI alterations will be analysed with focus on spontaneous progression or associated modifications after surgery. In addition, the quantity of intramedullary changes will be correlated to the severity of the cross-sectional spinal canal stenosis on the affected segment, as well as to the extension of clinical and electrophysiological restrictions.

\section{Statistical analysis}

We determined the group size of the prospective study based on the sensitivity of conventional T2w imaging. The reported sensitivity is approximately $60 \%$ whereby we estimated an improvement of the diagnostic accuracy to at least $80 \%$ based on our preliminary results. ${ }^{13}{ }^{14}$ To overcome potential problems based on biases derived from improved T2w imaging quality and potential over-interpretation of our preliminary results, we calculated the group size to reach a power of $90 \%$. Assuming a type I error of $5 \%$ and an improvement in sensitivity from $60 \%$ to $80 \%$ for our novel imaging method, the calculated sample size is 114 patients. Additionally, 20 healthy subjects should be measured as negative control to verify the principle of the new imaging method. The power analysis for our trial was evaluated in cooperation with the Department for Medical Biometrics and Statistics of the University of Freiburg.

Statistics are performed by IBM SPSS Statistics XXV and The R Project for Statistical Computing. The analysis includes the calculation of the sensitivity, specificity, positive and negative predictive value of the MIDI method to indicate functional myelopathy in comparison to the conventional T2w imaging. The correlations of clinical and electrophysiological factors to the several MIDI alterations and T2w signals are calculated through multiple regression analyses. All evaluations are done at inclusion and at the 12-month follow-up, whereas in functional symptom progression, the MIDI parameters will be screened for prognostic values.

\section{ETHICS AND DISSEMINATION}

The recruitment of patients started on 1st April 2018 and will be completed after approximately 24 months. Because of 12 months follow-up, the whole data acquisition receives at least 3 years. Patient and imaging data as well as the statistical analyses are available on a local server of the University Medical Center Freiburg respective international data security standards.

\section{Author affiliations \\ ${ }^{1}$ Department of Neurosurgery, Medical Center - University of Freiburg, Faculty of Medicine, University of Freiburg, Germany, Freiburg, Germany \\ ${ }^{2}$ Department of Neuroradiology, Medical Center - University of Freiburg, Faculty of Medicine, University of Freiburg, Germany, Freiburg, Germany \\ ${ }^{3}$ Department of Radiology, Medical Physics, Medical Center - University of Freiburg, Faculty of Medicine, University of Freiburg, Germany, Freiburg, Germany ${ }^{4}$ Department of Neurology, Medical Center - University of Freiburg, Faculty of Medicine, University of Freiburg, Germany, Freiburg, Germany}

Contributors $\mathrm{MH}$ and $\mathrm{KW}$ are responsible for the design and implementation of the study, as well as the following patient recruitment and data analysis. MH is the major contributor in writing the manuscript. KW additionally determined the electrophysiological evaluation. KE and MR are involved in the development, 
acquisition and post-processing of the imaging data. JHK and UH are mainly involved in determination of the clinical examinations and following patient recruitment. All authors read and approved the final manuscript.

Funding The study receives funding from the German Spine Society for the imaging and electrophysiological examinations. The article processing charge was funded by the German Research Foundation (DFG) and the University of Freiburg in the funding programme Open Access Publishing.

Competing interests None declared.

Patient consent for publication Not required.

Ethics approval The study protocol has been approved by the institutional ethics committee of the University of Freiburg, Germany (reference number 261/17).

Provenance and peer review Not commissioned; externally peer reviewed.

Open access This is an open access article distributed in accordance with the Creative Commons Attribution Non Commercial (CC BY-NC 4.0) license, which permits others to distribute, remix, adapt, build upon this work non-commercially, and license their derivative works on different terms, provided the original work is properly cited, appropriate credit is given, any changes made indicated, and the use is non-commercial. See: http://creativecommons.org/licenses/by-nc/4.0/.

\section{REFERENCES}

1. Bakhsheshian J, Mehta VA, Liu JC. Current diagnosis and management of cervical spondylotic myelopathy. Global Spine Journal 2017;7:572-86.

2. Nouri A, Tetreault L, Singh A, et al. Degenerative cervical myelopathy: epidemiology, genetics, and pathogenesis. Spine 2015;40:E675-93.

3. Fehlings MG, Arvin B. Surgical management of cervical degenerative disease: the evidence related to indications, impact, and outcome. $J$ Neurosurg 2009;11:97-100.

4. YL L. How has electrophysiology changed the management of cervical spondylotic myelopathy? Eur J Neurol 2008;15:781-6.

5. Simó M, Szirmai I, Arányi Z. Superior sensitivity of motor over somatosensory evoked potentials in the diagnosis of cervical spondylotic myelopathy. Eur J Neurol 2004;11:621-6.

6. Holly LT, Matz PG, Anderson PA, et al. Clinical prognostic indicators of surgical outcome in cervical spondylotic myelopathy. J Neurosurg 2009;11:112-8.

7. Matz PG, Anderson PA, Holly LT, et al. The natural history of cervical spondylotic myelopathy. J Neurosurg 2009;11:104-11.

8. Jutzeler CR, Ulrich A, Huber B, et al. Improved diagnosis of cervical spondylotic myelopathy with contact heat evoked potentials. $J$ Neurotrauma 2017;34:2045-53.

9. Meyer F, Börm W, Thomé C. Degenerative cervical spinal stenosis - current strategies in diagnosis and treatment. Dtsch Ärztebl Int 2008:366-72.

10. Martin AR, Aleksanderek I, Cohen-Adad J, et al. Translating stateof-the-art spinal cord MRI techniques to clinical use: a systematic review of clinical studies utilizing DTI, MT, MWF, MRS, and fMRI. Neuroimage 2016;10:192-238.

11. Matsuda Y, Miyazaki K, Tada K, et al. Increased Mr signal intensity due to cervical myelopathy. Analysis of 29 surgical cases. $J$ Neurosurg 1991;74:887-92.

12. Matsumoto $M$, Toyama $Y$, Ishikawa $M$, et al. Increased signal intensity of the spinal cord on magnetic resonance images in cervical compressive myelopathy. does it predict the outcome of conservative treatment? Spine 2000;25:677-82.

13. Yagi M, Ninomiya K, Kihara M, et al. Long-Term surgical outcome and risk factors in patients with cervical myelopathy and a change in signal intensity of intramedullary spinal cord on magnetic resonance imaging. J Neurosurg 2010;12:59-65.

14. Demir A, Ries M, Moonen CTW, et al. Diffusion-Weighted MR imaging with apparent diffusion coefficient and apparent diffusion tensor maps in cervical spondylotic myelopathy. Radiology 2003;229:37-43.

15. Witiw CD, Mathieu F, Nouri A, et al. Clinico-Radiographic discordance: an evidence-based commentary on the management of degenerative cervical spinal cord compression in the absence of symptoms or with only mild symptoms of myelopathy. Global Spine $J$ 2018:8:527-34

16. Tetreault L, Ibrahim A, Côté $P$, et al. A systematic review of clinical and surgical predictors of complications following surgery for degenerative cervical myelopathy. J Neurosurg 2016;24:77-99.

17. Vedantam A, Rajshekhar V. Does the type of T2-weighted hyperintensity influence surgical outcome in patients with cervical spondylotic myelopathy? A review. Eur Spine J 2013;22:96-106.

18. Suri A, Chabbra RPS, Mehta VS, et al. Effect of intramedullary signal changes on the surgical outcome of patients with cervical spondylotic myelopathy. The Spine Journal 2003;3:33-45.

19. Chatley A, Kumar R, Jain VK, et al. Effect of spinal cord signal intensity changes on clinical outcome after surgery for cervical spondylotic myelopathy. J Neurosurg 2009;11:562-7.

20. Takahashi M, Yamashita Y, Sakamoto Y, et al. Chronic cervical cord compression: clinical significance of increased signal intensity on $\mathrm{Mr}$ images. Radiology 1989;173:219-24.

21. Reisert M, Kellner E, Dhital B, et al. Disentangling micro from mesostructure by diffusion MRI: a Bayesian approach. Neuroimage 2017;147:964-75.

22. Wilson JR, Tetreault LA, Kim J, et al. State of the art in degenerative cervical myelopathy: an update on current clinical evidence. Neurosurgery 2017;80:S33-S45.

23. Ludolph ACet al. S1-Leitlinie Zervikale spondylotische Myelopathie. In: Leitlinien für Diagnostik und therapie in Der Neurologie. Deutsche Gesellschaft für Neurologie, 2017.

24. Zhong W, Liang X, Quan Z. Foot tapping test for lower extremity motor function of cervical spondylotic myelopathy. Zhong Nan Da Xue Xue Bao Yi Xue Ban 2014;39:296-300.

25. NURJCK S. The pathogenesis of the spinal cord disorder associated with cervical spondylosis. Brain 1972;95:87-100.

26. Kato S, Oshima $\mathrm{Y}$, Oka $\mathrm{H}$, et al. Comparison of the Japanese orthopaedic association (JOA) score and modified JOA (mJOA) score for the assessment of cervical myelopathy: a multicenter observational study. PLoS One 2015;10:e0123022.

27. Hufschmidt A, Lücking $\mathrm{CH}$, Rauer S. Neurologie compact - Für Klinik und Praxis. 5. Aufl. Stuttgart - New York: Georg Thieme Verlag, 2009.

28. Buchner H. Praxisbuch Evozierte Potenziale: Grundlagen, Befundung, Beurteilung und differenzialdiagnostische Abgrenzung. Aufl Georg Thieme Verlag 2014.

29. Kuhn F, Halder P, Spiess MR, et al. One-Year evolution of ulnar somatosensory potentials after trauma in 365 tetraplegic patients: early prediction of potential upper limb function. $J$ Neurotrauma 2012;29:1829-37.

30. Petersen JA, Spiess M, Curt A, et al. EM-SCI Study Group. spinal cord injury: one-year evolution of motor-evoked potentials and recovery of leg motor function in 255 patients. Neurorehabil Neural Repair 2012;26:939-48.

31. Kadanka Z, Adamova B, Kerkovsky M, et al. Predictors of symptomatic myelopathy in degenerative cervical spinal cord compression. Brain Behav 2017;7:e00797.

32. Kellner E, Dhital B, Kiselev VG, et al. Gibbs-ringing artifact removal based on local subvoxel-shifts: Gibbs-Ringing artifact removal. Magn Reson Med 2016;76:1574-81.

33. Veraart J, Novikov DS, Christiaens D, et al. Denoising of diffusion MRI using random matrix theory. Neuroimage 2016;142:394-406.

34. Kalsi-Ryan S, Karadimas SK, Fehlings MG. Cervical spondylotic myelopathy: the clinical phenomenon and the current pathobiology of an increasingly prevalent and devastating disorder. Neuroscientist 2013;19:409-21.

35. Fernández de Rota JJ, Meschian S, Fernández de Rota A, et al. Cervical spondylotic myelopathy due to chronic compression: the role of signal intensity changes in magnetic resonance images. $J$ Neurosurg Spine 2007;6:17-22. 